Conclusion This multidisciplinary intervention reduced antibiotic duration for lower respiratory tract infections and antibiotic related side-effects. This simple, effective intervention can be readily and quickly implemented into other clinical settings.

P24

\section{COMBATING INAPPROPRIATE USE OF ANTIBIOTICS THROUGH RAPID, ACCURATE AND COMPREHENSIVE DETECTION OF RESPIRATORY PATHOGENS USING A RESPIRATORY MULTIPLEX ARRAY}

doi:10.1136/thoraxjnl-2012-202678.165

'C Pollock, ${ }^{2} \mathrm{~J}$ McKenna, ' J O'Neill, 'R Thapliyal, 'IJ Lamont, 'S McKeown, 'M Crockard, ${ }^{2} \mathrm{P}$ Coyle, 'SP Fitzgerald. 'Randox Laboratories Limited, Crumlin, United Kingdom, ${ }^{2}$ Regional Virology Laboratory, Royal Victoria Hospital, Belfast

Introduction Respiratory tract infections (RTIs) are a leading cause of mortality and morbidity worldwide and one of the greatest causes of mortality in children under 5. Most people develop an acute RTI every year and these are the most common problems dealt with in primary care. RTI symptoms are similar but can be caused by a heterogeneous range of viral and bacterial pathogens.

Antibiotics were prescribed in $68 \%$ of acute respiratory tract visits and of those, $80 \%$ were unnecessary (CDC guidelines, 2001), with acute infection likely to be self-limiting. Repeated and improper use of antibiotics is the primary cause of increasing resistance, decreasing efficacy of these essential drugs. The best way to control resistance is to decrease antibiotic misuse by implementing rapid and accurate diagnosis, facilitating appropriate treatment.

A multiplex approach for detection of respiratory pathogens allows comprehensive screening, which is more effective in terms of time and resources compared to traditional methods thus allowing severely infected patients to be treated quickly and appropriately. This study reports the assessment of clinical samples using a respiratory multiplex array in a biochip platform for simultaneous detection of 22 clinically relevant bacterial and viral respiratory pathogens within a single patient sample (Table 1) and comparison against routine diagnostic approaches.

Methods Residual clinical respiratory samples $(n=502)$ were analysed using a Respiratory Multiplex Array applied to the Evidence Investigator analyser. This technology couples highly stringent multiplex PCR to biochip array technology. Results obtained were compared against routine diagnostic methods and \% agreement determined.

Results A high level of agreement was found between TaqMan based real-time assay and the Respiratory Multiplex Array. In a considerable number of samples, additional pathogens, previously unreported were detected with the Respiratory Multiplex Array. Sample re-testing confirmed that all were positive for the additional pathogens.

Conclusions These results highlight the relevance of multiplexing for detection of co-infections, enhancing patient care. The British Lung Foundation estimate respiratory illness costs NHS £6.6billion, so there is also potential for significant cost reductions should a system be implemented that allows for rapid and accurate detection of all causative agents of infection to ensure correct treatment.

Abstract P24 Table 1 Respiratory pathogens detected with the Respiratory Multiplex Array

\begin{tabular}{ll}
\hline Adenovirus & Influenza A \\
Bocavirus & Influenza B \\
Coronavirus 229E & RSV A \\
Coronavirus 0C43 & RSV B \\
Enterovirus & C. pneumoniae \\
Metapneumovirus & M. pneumoniae \\
Rhinovirus & L. pneumophila \\
Parainfluenza 1 & H. influenza \\
Parainfluenza 2 & Strep. pneumoniae \\
Parainfluenza 3 & Staph. aureus \\
\hline
\end{tabular}

\begin{tabular}{|l|l}
\hline P25 YEAR RETROSPECTIVE STUDY OF NON-TUBERCULOUS \\
\hline
\end{tabular} MYCOBACTERIA (NTM) ISOLATES

doi:10.1136/thoraxjnl-2012-202678.166

AJ Scadding, C Ohri, N Perera, G Woltmann. Glenfield Hospital, Institute of Lung Health University Hospitals of Leicester, Leicester, United Kingdom

Introduction The incidence non-tuberculous mycobacteria (NTM) has been growing steadily and was previously estimated between 2.9-4.43/100,000 persons. 125 NTM species have been identified due to advances in laboratory techniques with a corresponding increase of clinical presentations. Treatment is recommended if the same species is cultured on at least 2 occasions with clinical/radiological evidence for progression. Other than cystic fibrosis predisposing factors in adults include chronic lung disease, immunodeficiency, gastro-oesophageal reflux disease and postmenopausal non-smoking females. [1]

Methods 768 isolates were collected over 15 years in our regional mycobacterial database and analysed for demographic and frequency distributions using simple statistics. The data includes many single, incidental isolates as well as patients with multiple isolates and on-going symptoms.

Results We analysed 768 non-tuberculous mycobacterial cultures from 534 patients. The mean age for first positive culture was 55 (range $0-102$ ) with $42.7 \%$ of the isolates occurring above age 65 . $58 \%(310)$ of subjects were male, $61.3 \%$ were white Caucasian and $24.5 \%$ were of South Asian ethnicity. $81.4 \%$ of isolates were single with only $2 \%$ of patients culturing more than 4 isolates. The patient with most isolates (12) grew Mycobacterium Avium Complex (MAC) repeatedly over a 10-year time span. The most common isolate was MAC (37.0\%) followed by the rapidly growing mycobacteria (RGM) which accounted for $28.4 \%$. In descending order M. Kansasii, M. Gordonae and M. Xenopi accounted for 8.7\%, 6.9\% and $3.9 \%$ of the isolates respectively. The majority of cultures were of respiratory origin from sputum $(76.2 \%)$ and broncho-alveolar lavage $(7 \%)$ samples. $3.8 \%$ of samples were cultured from pus, $1.3 \%$ from blood cultures and $0.7 \%$ from lymph nodes.

Discussion In keeping with previous studies MAC was the most commonly identified mycobacterium and the most common population were older, white peoples. Our data for culture site compares to that published by the American Centers for Disease Control and Prevention. Our data highlights that a variety of NTM can be isolated from multiple locations and pose a diagnostic challenge.

1. DE Griffith et al., "An Official ATS/IDSA Statement: Diagnosis, Treatment, and Prevention of Nontuberculous Mycobacterial Diseases," AJRCCM 175, no.4 (February 2007): 367-416.

\section{Management and organisation of respiratory health care}

\section{P26 RESPIRATORY PATIENT PREFERENCE AND SATISFACTION RAPIDLY EVALUATED WITH TABLET PC USING THE 18-ELEMENT CONSULTATION SPECIFIC OUESTIONNAIRE (CSO): COMPARISON WITH SURGICAL PATIENTS}

doi:10.1136/thoraxjnl-2012-202678.167

J Beeston, AK Datta. York Teaching Hospital, Hull York Medical School, York, UK

Patient perspective is important for quality assessment of service offered by hospitals and individual clinicians, evidenced by annual surveys required by the Care Quality Commission for all English NHS Acute Trusts. Patient satisfaction has been measured in consultations in Primary Care ${ }^{1}$. However as yet unanswered, is which aspects of consultations do patients value most, and whether this preference is dependent on patient type and mode of presentation. We used a tablet PC (Customer Research Technologies) or paper 